\title{
The comparison of short-term postoperative outcomes between upfront surgery and surgery following neoadjuvant treatment in pancreatic ductal adenocarcinoma
}

\author{
Ji Hye JUNG, So Kyung YOON, So Jeong YOON, Sang Hyun SHIN, In Woong HAN, Jin Seok HEO* \\ Division of Hepatobiliary-Pancreatic Surgery, Department of Surgery, Samsung Medical Center, \\ Sungkyunkwan University School of Medicine, Seoul, Korea
}

Introduction: In cases that neoadjuvant treatment (NAT) was performed before surgery, research on short-term postoperative outcomes seems to have been insufficient. We aimed to compare short-term outcomes between patients who received upfront surgery and those who received surgery following NAT for pancreatic ductal adenocarcinoma (PDAC).

Methods: We retrospectively reviewed 1,244 cases of PDAC that had pancreatectomy at Samsung Medical Center from January 2010 to March 2020. The resectability status was evaluated according to NCCN guidelines 2017, and cases with distant metastasis were excluded. All cases were classified into two groups of resectable group (R group) and borderline resectable group (BR group). In each group, factors were compared between the cases that upfront surgery was performed (UpS cases) and the cases that surgery was performed after NAT (NAT cases).

Results: In R group ( $\mathrm{n}=981)$, the clinical, operative, pathologic features did not show distinct differences between UpS cases $(\mathrm{n}=973)$ and NAT cases $(n=8)$. In BR group $(n=248)$, patients were younger, preoperative/pre-NAT tumor size was larger, and less cases were accompanied by vascular resection in NAT cases $(n=85)$ compared with UpS cases $(n=163)$. There were many oncologic benefits in BR group compared with R group. The short-term postoperative outcomes had no significant differences between UpS cases and NAT cases in both R group and BR group.

Conclusions: In cases of NAT, there were no significant differences from upfront surgery in terms of short-term outcomes including postoperative complication. Therefore, we think it is reasonable to perform aggressive surgery for NAT patients. 